\title{
ESA-led ATHENA/IXO optics development status
}

Bavdaz, Marcos; Rando, Nicola; Wille, Eric; Wallace, Kotska; Shortt, Brian; Collon, Maximilien; van Baren, Coen; Pareschi, Giovanni; Christensen, Finn Erland; Krumrey, Michael

Total number of authors:

11

Published in:

Proceedings of SPIE - The International Society for Optical Engineering

Link to article, DOI:

$10.1117 / 12.893567$

Publication date:

2011

Document Version

Publisher's PDF, also known as Version of record

Link back to DTU Orbit

Citation $(A P A)$ :

Bavdaz, M., Rando, N., Wille, E., Wallace, K., Shortt, B., Collon, M., van Baren, C., Pareschi, G., Christensen, F. E., Krumrey, M., \& Freyberg, M. (2011). ESA-led ATHENA/IXO optics development status. Proceedings of SPIE - The International Society for Optical Engineering, 8147(1), 81470C. https://doi.org/10.1117/12.893567

\section{General rights}

Copyright and moral rights for the publications made accessible in the public portal are retained by the authors and/or other copyright owners and it is a condition of accessing publications that users recognise and abide by the legal requirements associated with these rights.

- Users may download and print one copy of any publication from the public portal for the purpose of private study or research.

- You may not further distribute the material or use it for any profit-making activity or commercial gain

- You may freely distribute the URL identifying the publication in the public portal 


\title{
ESA-led ATHENA/IXO Optics Development Status
}

\author{
Marcos Bavdaz ${ }^{1}$, Nicola Rando ${ }^{1}$, Eric Wille ${ }^{1}$, Kotska Wallace ${ }^{1}$, Brian Shortt ${ }^{1}$, Maximilien Collon ${ }^{2}$, \\ Coen van Baren ${ }^{3}$, Giovanni Pareschi ${ }^{4}$, Finn Christensen ${ }^{5}$, Michael Krumrey ${ }^{6}$, Michael Freyberg ${ }^{7}$ \\ ${ }^{1}$ European Space Agency, ESTEC, Keplerlaan 1, PO Box 299, NL-2200 AG Noordwijk, The Netherlands \\ ${ }^{2}$ cosine Research B.V, Niels Bohrweg 11, NL-2333 CA Leiden, The Netherlands \\ ${ }^{3}$ SRON, Sorbonnelaan 2, 3584 CA Utrecht, The Netherlands \\ ${ }^{4}$ Osservatorio Astronomico di Brera, Via E. Bianchi 46 I- 23807, Merate (Lc), Italy \\ ${ }^{5}$ DTU-space, Juliane Maries Vej 30, DK-2100 Copenhagen, Denmark \\ ${ }^{6}$ Physikalisch-Technische Bundesanstalt (PTB), Abbestr. 2-12, D-10587 Berlin, Germany \\ ${ }^{7}$ MPI f. extraterrestrische Physik, Giessenbachstrasse 1, D-85748 Garching, Germany
}

\begin{abstract}
The International X-ray Observatory (IXO) is a candidate mission in the ESA Space Science Programme Cosmic Vision 1525 , and was studied as a joint mission with NASA and JAXA. Considering the programmatic evolution of the international context, the mission is being reformulated as an ESA-led mission, under the name of ATHENA (Advanced Telescope for High Energy Astrophysics), with possible participation of NASA and JAXA.

The mission is building on the novel Silicon Pore Optics (SPO) technology to achieve the required performance for this demanding astrophysics observatory. This technology is being developed by an industrial consortium, and involves also several research institutes [1-12]. A second optics technology, slumped glass optics (SGO), which is being developed in Europe and the USA, was the backup technology for IXO, and additionally work is progressing on improved reflective coatings and X-ray test facilities [13-17].
\end{abstract}

Keywords: X-ray optics, X-ray astronomy, ATHENA, IXO, pore optics, X-ray telescopes, X-ray testing

\section{INTRODUCTION}

ATHENA is the result of the IXO reformulation activities, started at the end of March 2011. The reformulation was initiated by ESA in consequence of the evolution of the international cooperation scenario of IXO (International X-ray Observatory) in March 2011, which prevents the implementation of such a mission within the timescale and budget previously envisaged. As it is well known, IXO was the result of the merger of the XEUS and Constellation-X study activities, agreed by ESA, JAXA and NASA in 2008 [18-22].

ATHENA is now a candidate L-class missions in the Cosmic Vision plan for the 2022 launch slot, and is undergoing industrial studies in preparation of a competitive selection process. The definition studies will start in 2012, and the final adoption of one L-class mission for flight implementation is expected in 2014.

The programmatic constraints for ATHENA are driven by the budget envelope of the ESA Science and Robotic Exploration directorate and by the implementation schedule needs. The general requirements for the L-class mission are to secure the 2022 launch date and to maintain ESA Cost at Completion $(\mathrm{CaC})$ within the assigned limits.

ATHENA is a facility-class mission that will address the leading astrophysical questions in the "hot universe" through its breakthrough capabilities in X-ray spectroscopy, imaging and timing. The high level science requirements are listed below in table 1 .

Optics for EUV, X-Ray, and Gamma-Ray Astronomy V, edited by Stephen L. O'Dell, Giovanni Pareschi, Proc. of SPIE Vol. 8147, 81470C · C 2011 SPIE · CCC code: 0277-786X/11/\$18 - doi: 10.1117/12.893567 
Table 1: Science Requirements developed as an input to the internal design study of ATHENA.

\begin{tabular}{|c|c|c|}
\hline $\begin{array}{l}\text { Effective Area } \\
\text { (total) }\end{array}$ & $0.5 \mathrm{~m}^{2} @ 6 \mathrm{keV}$ (goal 0.7) & $\begin{array}{l}\text { Black hole evolution, large scale structure, cosmic } \\
\text { feedback, } \\
\text { Strong gravity, Equation of state }\end{array}$ \\
\hline $\begin{array}{l}\text { Spectral } \\
\text { Resolution } \\
\text { (FWHM) }\end{array}$ & $\begin{array}{c}\Delta \mathrm{E}=3 \mathrm{eV}(\mathrm{G} 6 \mathrm{keV}) \text { within } 2 \times 2 \mathrm{arc} \min \text { (goal of } \\
2.5 \mathrm{eV} \text { and } 3 \times 3 \operatorname{arc~min}) \\
\Delta \mathrm{E}=150 \mathrm{eV} \text { at } 6 \mathrm{keV} \text { within } 25 \mathrm{arc} \text { min diam (goal } \\
\text { of } 125 \mathrm{eV} \text { and } 30 \mathrm{arc} \mathrm{min} \text { ) }\end{array}$ & $\begin{array}{l}\text { Black Hole evolution \& cosmic feedback } \\
\text { Large scale structure }\end{array}$ \\
\hline $\begin{array}{l}\text { Angular } \\
\text { Resolution }\end{array}$ & $\begin{array}{c}10 \operatorname{arc~sec~HPD~}(0.1-7 \mathrm{keV}) \\
\text { goal of } 5 \operatorname{arc~sec}\end{array}$ & $\begin{array}{l}\text { Large scale structure, cosmic feedback, black hole } \\
\text { evolution, missing baryons }\end{array}$ \\
\hline $\begin{array}{l}\text { Count Rate } \\
\text { (WFI) }\end{array}$ & $\begin{array}{c}1 \text { Crab with }>90 \% \text { throughput (tbc) } \\
\Delta \mathrm{E}<200 \mathrm{eV} @ 6 \mathrm{keV}(0.3-10 \mathrm{keV})\end{array}$ & $\begin{array}{l}\text { Strong gravity } \\
\text { Equation of state }\end{array}$ \\
\hline Astrometry & $1.5 \operatorname{arcsec}$ at $3 \sigma$ confidence $(t b c)$ & Black hole evolution \\
\hline Absolute Timing & $100 \mu \mathrm{sec}$ & Neutron star studies \\
\hline
\end{tabular}

\section{THE ATHENA BASELINE DESIGN}

The baseline mission scenario selected for ATHENA is a large halo orbit at the Sun/Earth-L2, reached with an Ariane 5/ECA (A5) launch. The thermal environment is exceptionally stable, offering ideal conditions for the thermal control system of the detector instruments and of the telescope mirror assembly (MA).

The ATHENA telescope reference design is based on a focal length $(\mathrm{F})$ of $11.5 \mathrm{~m}$, consisting of two units, each comprising of about 250 Silicon Pore Optics Mirror Modules (SPO MM). In order to optimize the utilization of the available space, the outer rows of the telescope units are only partially filled, exploiting the modular nature of the Silicon Pore Optics. A sketch of the design is depicted in figure 1.
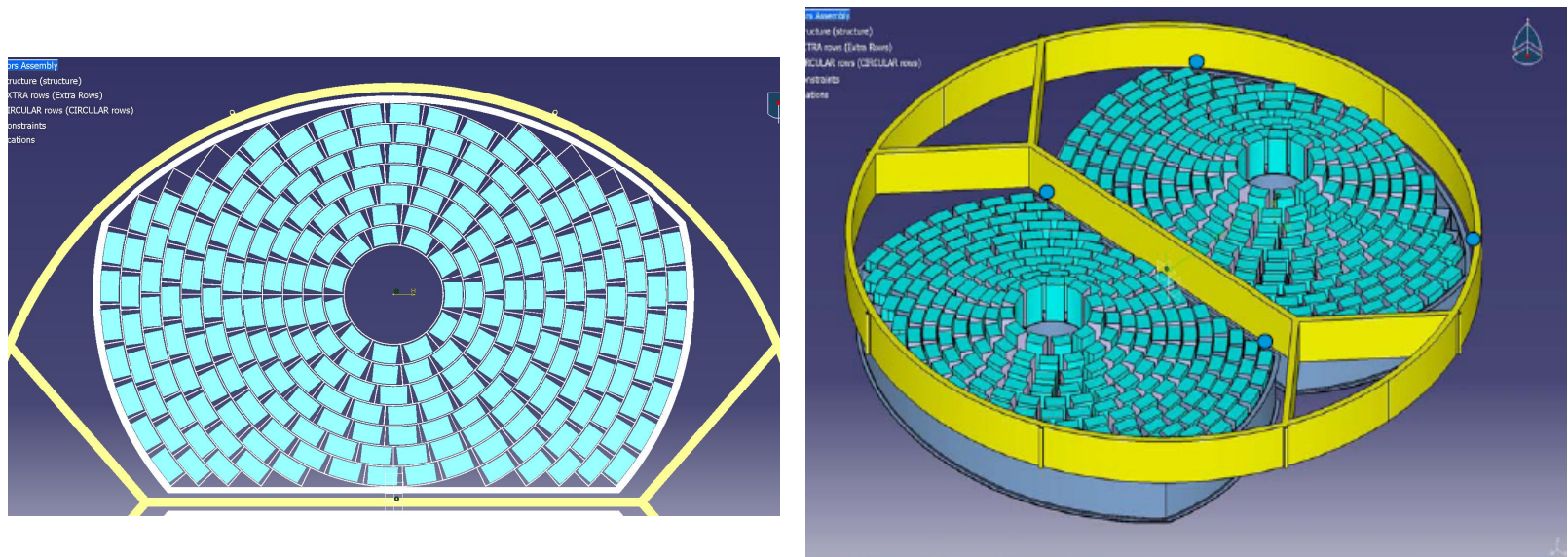

Figure 1: A sketch of the ATHENA telescope concept, due to its appearance referred to as the "owl design". On the left the population of the telescope aperture with the Silicon Pore Optics Mirror Modules (SPO MM) is shown. Benefit is taken of the modularity of this technology to efficiently tile the area provided by the spacecraft structure. On the right a 3-D view is shown, with the SPO MM displayed in cyan, mounted on petal structures indicated in grey, and including the support structure, indicated in yellow. 
In the reference design, the ATHENA telescope inner-most radius is $0.15 \mathrm{~m}$, with the outer-most extending to $0.58 / 0.90$ $\mathrm{m}$, depending on the azimuth. The estimated effective area at $1.25 \mathrm{keV}$ is $1.18 \mathrm{~m}^{2}$, and at $6 \mathrm{keV}$ it is $0.55 \mathrm{~m}^{2}$, fulfilling the science requirements as outlined in table 1.

The science objectives of the Athena mission can be fulfilled with a payload complement consisting of a cryogenic X-ray microcalorimeter (XMS) and a Wide Field Imager (WFI). The instruments are located on the focal plane assembly (FPA), separated from their dedicated telescope units through a fixed metering structure. The baseline spacecraft configuration therefore resembles the XMM-Newton design, see figure 2.

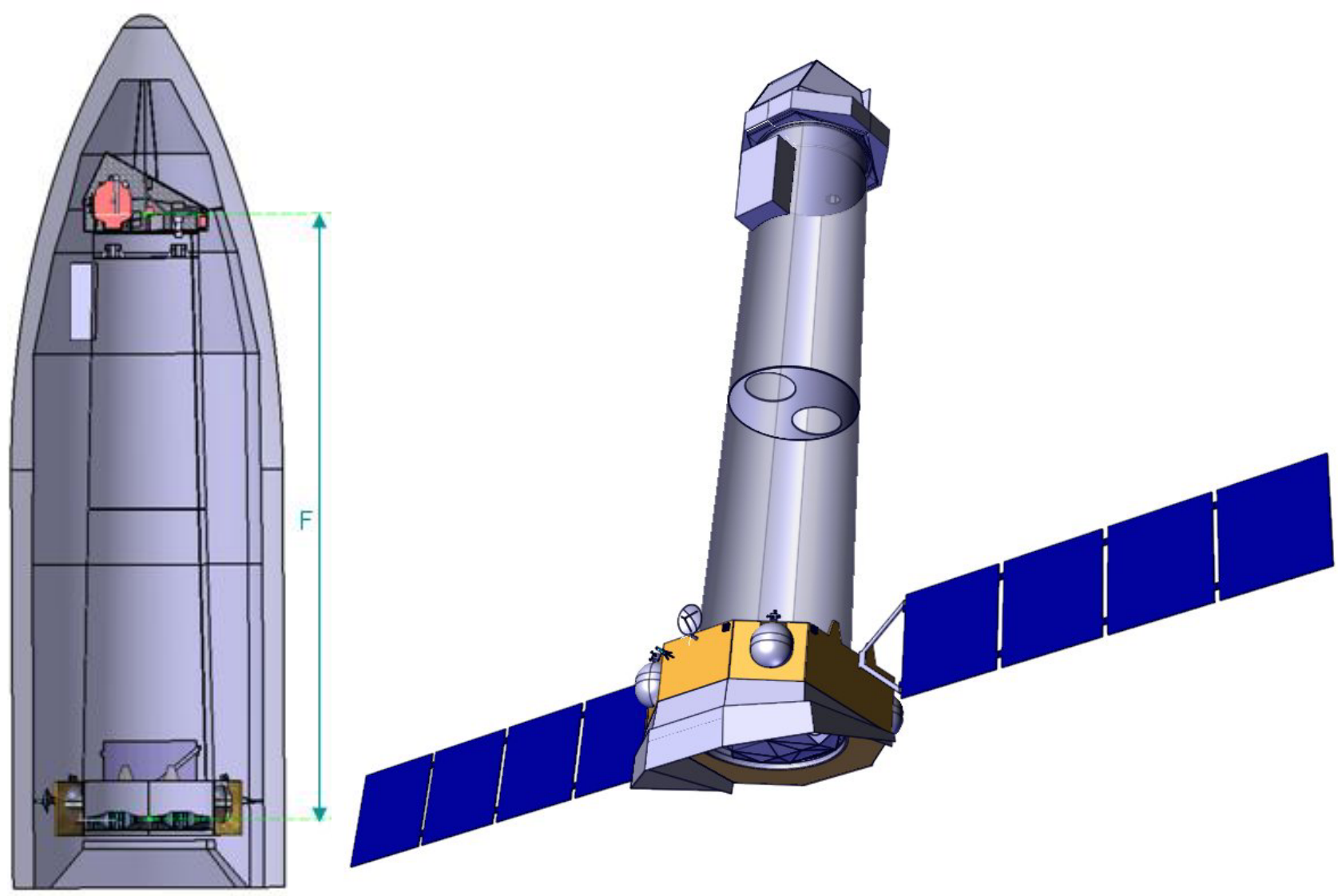

Figure 2: The ATHENA spacecraft configuration is much simplified with respect to the IXO configuration. On the left ATHENA is shown as stowed for launch inside the A5 fairing, and at the right as deployed in the operational orbit. A fixed optical bench is used, providing a focal length $\mathrm{F}$ of about $11.5 \mathrm{~m}$. The two detector instruments have dedicated telescope units, which are co-aligned. No detector exchange mechanism is therefore required.

Based on the preliminary internal study the ATHENA spacecraft would have a total mass of just above 4 tons, including all required margins. The total estimated power budget (EOL) is estimated to be about $5 \mathrm{~kW}$. The total mass of the two focal plane instruments, including design maturity margins is of about $470 \mathrm{~kg}$. The total mass of the Silicon Pore Optics is about $330 \mathrm{~kg}$. 


\section{THE ATHENA OPTICS TECHNOLOGY}

The Silicon Pore Optics technology is the baseline for the ATHENA telescope. This technology has been introduced for the XEUS mission candidate, and then became the baseline technology for the IXO mission, which defined the boundary conditions and requirements for the technology development activities. The move to ATHENA essentially has the following implications on the telescope optics technology:

- Focal length: $20 \mathrm{~m} \rightarrow 11.5 \mathrm{~m}$

- Inner-most radius: $0.3 \mathrm{~m} \rightarrow 0.15 \mathrm{~m}$

- Outer-most radius: $1.7 \mathrm{~m} \rightarrow 0.9 \mathrm{~m}$

- Operating temperature: closer to room temperature

- Updated thermal gradient and mechanical load cases expected following system studies

- Number of Mirror Module (MM): $\sim 1700 \rightarrow \sim 500$

- Number of petals per telescope: $8 \rightarrow 1$

- Angular resolution: 10" HEW requirement (and 5"goal)

The ATHENA requirements simplify the SPO technology developments in most areas. The shorter focal length requires smaller test facilities and shorter mirror plates, and the operating temperature is increased to 20 degrees $\mathrm{C}$ with reduced thermal gradients. A lower number of mirror units is required, facilitating the flight model production, and the accommodation is likely accomplished on only one petal per telescope.

Since for the current design of ATHENA the optics are populated down to radii of $0.15 \mathrm{~m}$, some mirror modules will be needed, which are outside the parameter space envisaged for IXO. In table 2 the values of some key parameters (mirror plate bending radius, mirror plate length, and the wedge height required to achieve the con-focal arrangement of the mirror shells) are given for the IXO configuration, the technology demonstrator used until now, and the ATHENA configuration. The later lists two cases: (left) the main area down to a radius of $0.25 \mathrm{~m}$, and (right) the innermost one or two rows to $0.15 \mathrm{~m}$ radius. Note that an inner radius of $0.25 \mathrm{~m}$ would be sufficient in case the focal length of the ATHENA telescope can be extended to $12 \mathrm{~m}$ - this is a subject of ongoing system studies.

Table 2: SPO Mirror Plate parameters for IXO, the technology demonstrator mirror module and ATHENA: Except for the innermost mirror modules, the ATHENA mirror modules can be produced using the same manufacturing technology as for IXO.

\begin{tabular}{|l|l|l|l|l|}
\hline & IXO (f=20m) & Demonstrator (2011) & \multicolumn{2}{|l|}{ ATHENA (f=11.5 m) } \\
\hline Radius $[\mathrm{m}]$ & $0.25-1.75$ & 0.74 & $0.25-0.9$ & $(0.15)$ \\
\hline Plate Length $[\mathrm{mm}]$ & $28-194$ & 67 & $30-111$ & $(186)$ \\
\hline Wedge height $[\mu \mathrm{m}]$ & $0.27-5.6$ & $0.63 \& 1.9$ & $0.52-5.6$ & $(3.1 \& 9.4)$ \\
\hline
\end{tabular}

The current mirror module demonstrator and the IXO parameter studies already cover the needs of ATHENA down to a radius of $0.25 \mathrm{~m}$, i.e. the ATHENA mirror modules for $\mathrm{R}>0.25 \mathrm{~m}$ can be manufactured using the same SPO manufacturing technology as foreseen for IXO. For the innermost mirror modules the bending radius has to be smaller, and the wedge angle and associated height for the second reflector has to be larger than explored in detail to date. The Technology Development Plan for the ATHENA optics is therefore currently being updated to include also these extensions.

To gain confidence in the ability to produce also the innermost mirror modules of ATHENA, tests have been performed to determine the minimum possible bending radius for the mirror plates as used for the mirror module demonstrator. The mirror plates are designed for a radius of curvature of $0.74 \mathrm{~m}$, representative of the average IXO and ATHENA mirror module. Ignoring the fact, that, for the inner radii the mirror plate design has yet to be optimised, including the implementation of a thinner and more flexible mirror membrane, the test was conducted successfully. A bending radius of less than $0.06 \mathrm{~m}$ could be demonstrated with the rather thick standard plates, see figure 3 . 


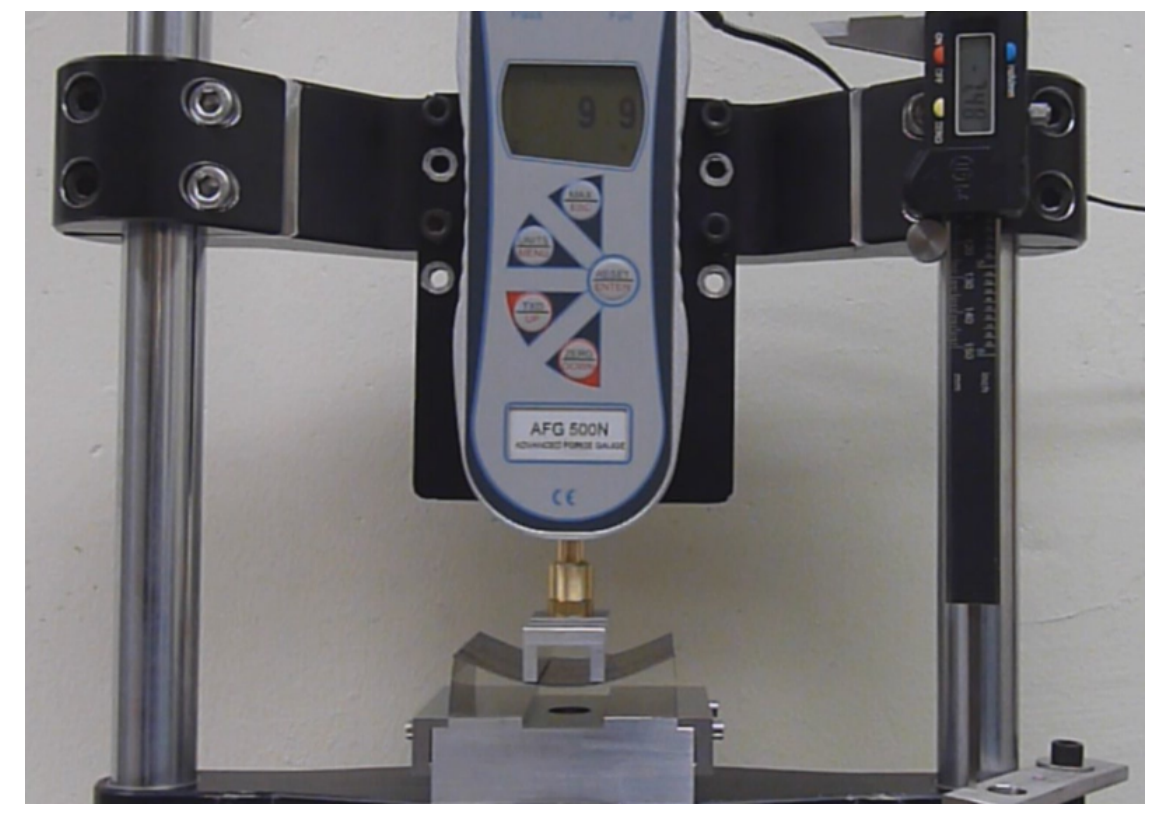

Figure 3: Mirror plate bending test demonstrating the ability to bend standard mirror plates (i.e. not optimised for the inner radii) to a radius of less than $0.06 \mathrm{~m}$ without breakage. This bending radius is 2.5 times smaller than required for the innermost mirror plate of ATHENA. During the test the displacement and the applied force were recorded. For the actual inner mirror modules optimised mirror plates are foreseen, featuring an even better flexibility and lower bending strain.

\section{THE ATHENA OPTICS TECHNOLOGY DEVELOPMENTS}

The SPO technology development plan (TDP) developed for IXO remains largely valid, and the implementation of the individual technology activities continues. The plan is currently being reviewed and updated considering the specific needs of ATHENA. The definition of new activities primarily addresses the innermost mirror modules, and in addition modifications to the test facilities are being discussed to accommodate the focal length of ATHENA. For the focal length of ATHENA the conical approximation becomes more dominant than it was for IXO: the contribution in the HEW error budget increases from 3.1 arcsec for $20 \mathrm{~m}$ focal length, to 5.4 arcsec at $11.5 \mathrm{~m}$. The introduction of a true Wolter design or a derivative thereof was already planned in the IXO TDP, being addressed by a dedicated technology activity; its relevance is increased in the case of ATHENA.

The main priorities of the technology development activities are directed to the demonstration of:

- the compatibility with the environmental requirements (ruggedisation)

- the achievement of the required angular resolution (10" HEW)

- the feasibility to produce the flight model optics within budget and on schedule (mass production)

Additional activities regard the required test facilities (both pencil beam scanning using synchrotron radiation, and full area illumination at the Panter facility), and mirror plate coatings (metal layers with over-coatings and multi-layers). Further, the development activities regarding the European glass optics also continue.

A milestone was achieved with the vibration tests of an SPO mirror module in 2010. The test was conducted by KayserThrede at the facilities of SGS Germany (a test house located in Munich), and involved tests in the two major axis of the mirror module (X-direction normal to the optical axis and parallel to the mirror surface, and Z-direction parallel to the optical axis). The goal of vibration tests was the validation of the finite element models of the mirror module, and the determination of the actual resonance frequencies and gain factors. Before and after the vibration tests X-ray metrology was performed to verify that no changes occurred to the optical performance. In figure 4 the set-up is shown, for the Z- 
configuration, and showing the 3-axis accelerometers mounted on several locations on each of the mirror plate stacks, the bracket plates and the support jig. The FEM could be confirmed, and in fact the measured Q-factor was lower than anticipated. The resonance frequencies were close to the calculated values, and the deviations served to tune the numerical models of the mirror unit.
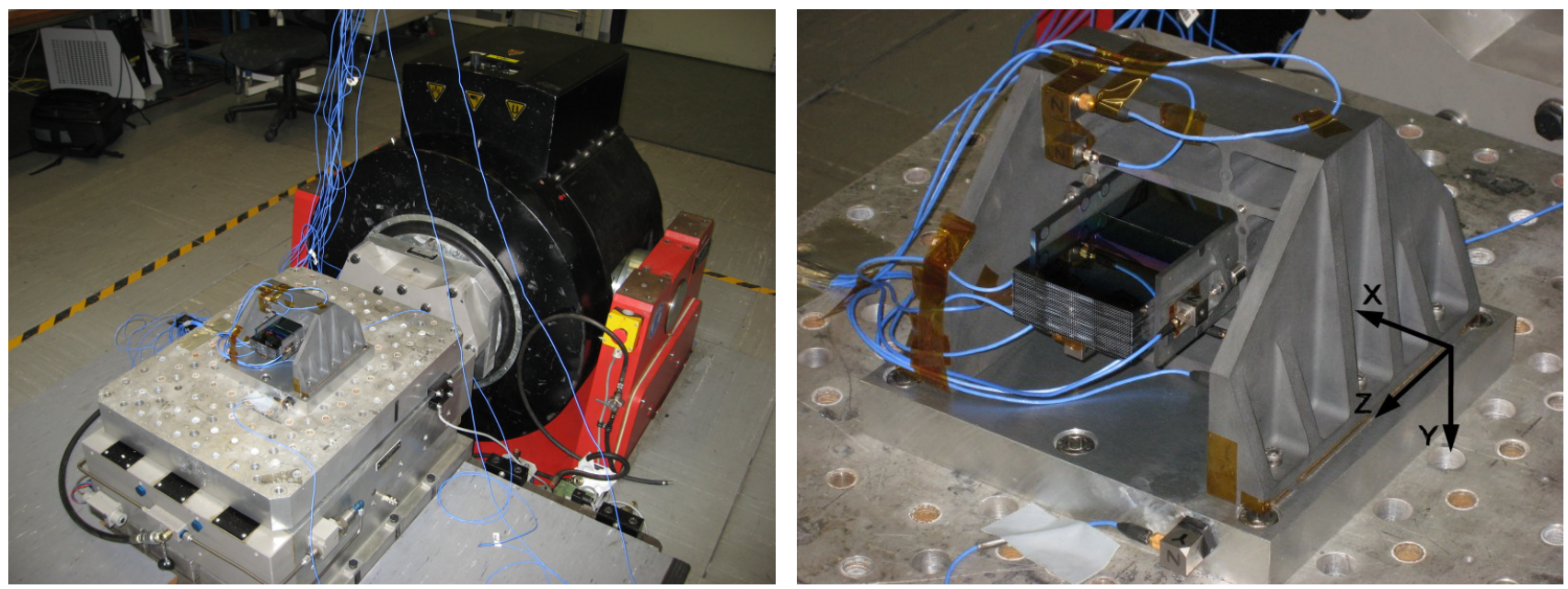

Figure 4: Vibration tests of mirror module to validate the numerical modelling of the unit. All resonances were found to be well above the requirements $(200 \mathrm{~Hz})$, and the measured Q-factor was 21 (FEM models assumed $\mathrm{Q}=25$, i.e. they were conservative).

The angular resolution performance of the SPO modules is further improving. Two independent X-ray facilities (BESSY II and Panter) are used to measure the point spread function and the angular resolution of the optics. In figure 5 two recent mirror modules are shown. The unit on the left (XOU7) shows a HEW of $7.5 \operatorname{arcsec}$ for the complete area of the first 4 mirror plates (indicated by the red rectangle), and the unit on the right (XOU8) demonstrated a HEW of 16.6 arcsec on the complete area of the mirror module, consisting of 45 mirror plates.
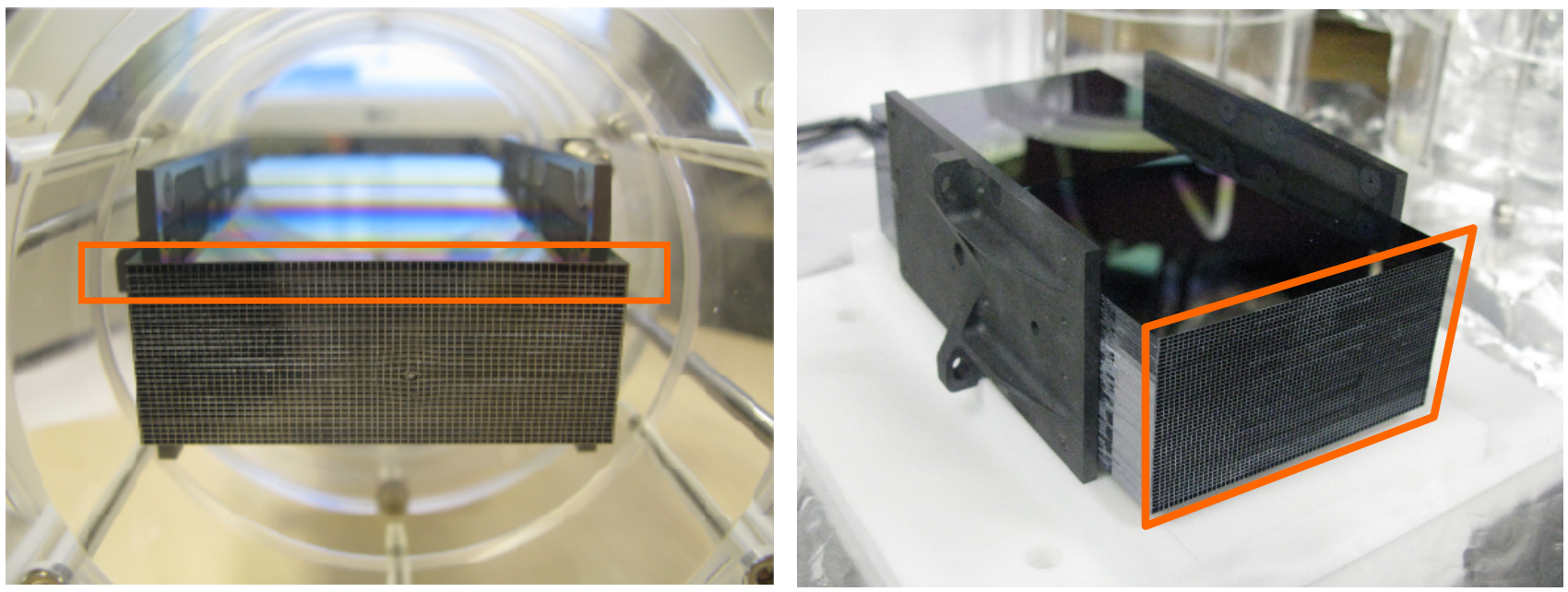

Figure 5: SPO mirror module on the left (XOU7) demonstrated a measured X-ray HEW of 7.5 arcseconds over the complete area of the first 4 mirror plates. The mirror module on the right (XOU8), consisting of 45 mirror plates measured 16.6 arcseconds for the complete areas of the module. Both values are double reflection, measured with X-rays at $3 \mathrm{keV}$. The red rectangles indicate the measured area. 
A new robotic system to produce mirror stacks has been developed by cosine Research and brought on line, and the commissioning was successfully concluded. The new system has some important improvement over the robotic system used to assemble the mirror modules shown in figure 6. Employing a rigid hexapod manipulator for positioning the stack to the mirror plates, and a newly developed surface figure metrology system, this equipment is the prototype of the machines to be used for the later mass production of the flight model optics.

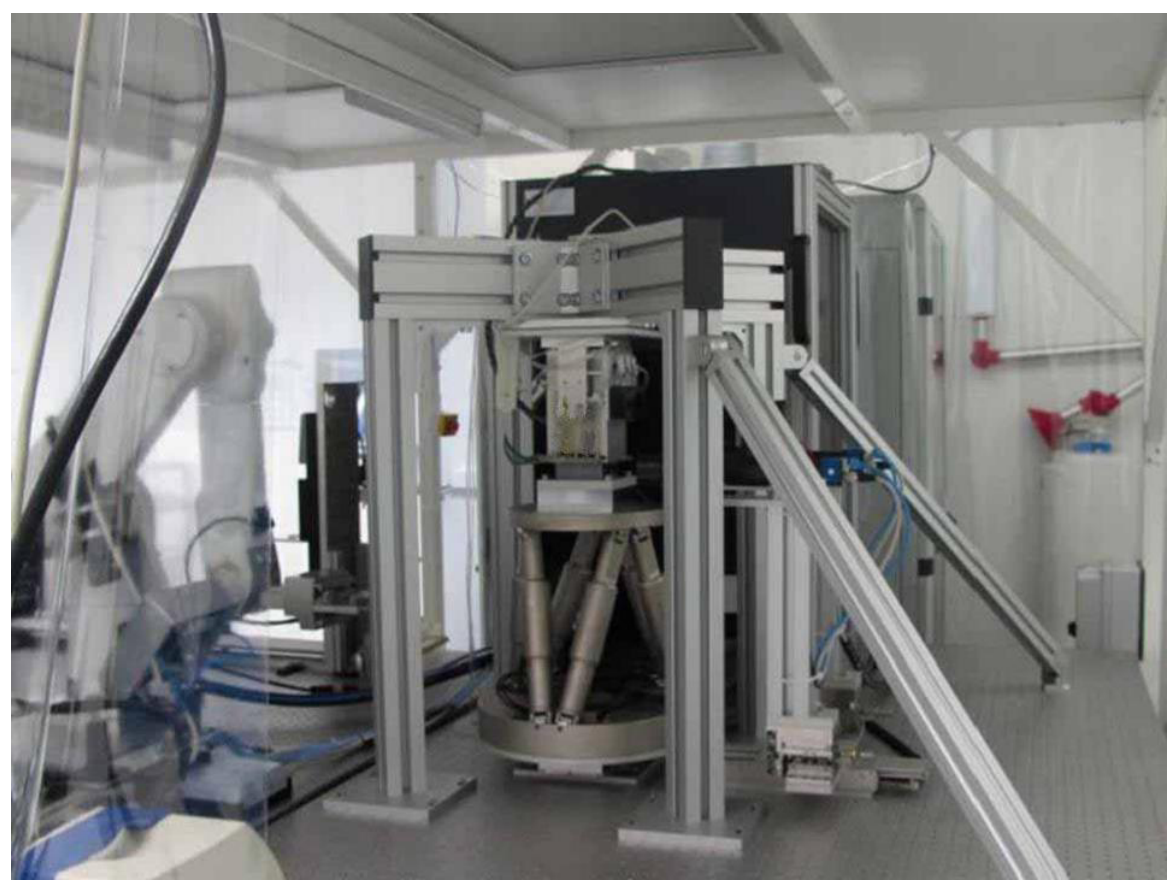

Figure 6: A new stacking robot was built by cosine Research and brought on-line, implementing several improvements over the system used in the past. Being much stiffer and employing a newly developed forming tool and surface figure metrology system, it is based on the architecture planned for the equipment to be used to produce the flight model mirror stacks for ATHENA.

In addition, a laser labelling system was introduced, which marks each individual mirror plate with a unique code, making it possible to trace each mirror plate in a mirror module back to the wafer used to manufacture it. The mirror plates also undergo an upgraded cleaning process and are automatically checked with an improved particle inspection system prior to stacking.

\section{CONCLUSION}

ATHENA has emerged from the re-formulation of IXO, required by ESA in consequence of the evolution of the international cooperation scenario. ATHENA is now a candidate L-class mission in the Cosmic Vision plan for the 2022 launch slot.

The conventional and robust mission design of ATHENA represents a significant simplification with respect to IXO. No new technology developments are required on the spacecraft side. Nevertheless, ATHENA is a very powerful observatory with a performance compliant to the science requirements. The development risk is drastically reduced by reducing the number of focal plane instruments to two, a simplified cryogenic spectrometer and a wide field imager. For the optics, the relaxation of the nominal angular resolution requirement and energy bandwidth, together with (near) room temperature operation, brings the SPO technology closer to flight readiness. 
The ATHENA optics technology is the Silicon Pore Optics (SPO), and only minor modifications are required to the related technology development activities.

Progress has been achieved in all three priority areas of the optics developments: ruggedisation, improved performance and mass production. These accomplishments are the result of executing a long-term-vision technology development plan. This plan is now being updated to include the needs of ATHENA and will ensure the timely availability of the Silicon Pore Optics.

\section{ACKNOWLEDGEMENTS}

A large group of institutions and companies, as well as individuals have to be mentioned and acknowledged. Without their solid commitment and engagement in their contributions to the developments presented here, the technology preparation of ATHENA would not be progressing so swiftly.

Beyond the authors, major contributors were: from cosine Research B.V (The Netherlands): Marcelo Ackermann, Ramses Günther, Rakesh Partapsing, Giuseppe Vacanti, Marco W. Beijersbergen; from Micronit (The Netherlands): Mark Olde Riekerink, L. de Vreede, B. Lansdorp, M. Blom; from Kayser-Threde (Germany): Markus Erhard, C. Körner, Dirk Kampf; from Stichting Ruimte-Onderzoek Nederland (The Netherlands): Coen van Baren; from Denmarks Tekniske Universitet (Denmark): Anders Jakobsen, Desiree Della Monica Ferreira, Carsten Jensen, Finn Christensen; from Max-Planck-Institut für extraterrestrische Physik (Germany): Michael Freyberg, Wolfgang Burkhard; from Physikalisch-Technische Bundesanstalt (Germany): Peter Müller, Levent Cibik.

For the IXO back-up optics: from Osservatorio Astronomico di Brera (Italy): Mauro Ghigo, Stefano Basso, Paolo Conconi, Oberto Citterio, Marta Civitani, Laura Proserpio, Daniele Spiga, Gianpiero Tagliaferri, Alberto Zambra; from Max-Planck-Institut für extraterrestrische Physik (Germany): Peter Friedrich, Monika Vongehr, Anita Schael.

\section{REFERENCES}

1. M. Beijersbergen et al., Proc SPIE 5539, (2004)

2. M. Bavdaz et al, Proc SPIE 5488, (2004)

3. M. Bavdaz et al, Proc SPIE 5900, (2005)

4. M. Beijersbergen et al, American Astronomical Society Meeting Abstracts, volume 213 ( 2009)

5. O. Riekerink et al, Proc. of SPIE Vol. 7437 (2009)

6. K. Wallace et al, Proc. of SPIE Vol. 7437 (2009)

7. M. Collon et al, Proc. of SPIE Vol. 7437 (2009)

8. D. Kampf et al, Proc. of SPIE Vol. 7437 (2009)

9. M. Ackermann et al, Proc. SPIE 7437, (2009)

10. M. Bavdaz et al, Proc of SPIE 7732, (2010)

11. M. Collon et al, Proc of SPIE 7732, (2010)

12. M. Ackermann et al, Proc of SPIE 7732, (2010)

13. M. Ghigo et al, Proc of SPIE 7732, (2010)

14. M. Civitani et al, Proc of SPIE 7732, (2010)

15. M. Ackermann et al, Proc of SPIE 7732, (2010)

16. M. Krumrey et al, Proc of SPIE 7732, (2010)

17. W. Zhang et al, Proc of SPIE 7732, (2010)

18. A. Parmar, G. Hasinger and M. Turner Proc $34^{\text {th }}$ Cospar Assembly, (2004)

19. J. Bookbinder et al, Proc. of SPIE 7011, (2008)

20. M. Bavdaz et al, Proc of SPIE 7437, (2009)

21. J. Bookbinder, Proc of SPIE 7732, (2010)

22. N. Rando et al, Proc of SPIE 7732, (2010) 